\title{
4DMRI-based investigation on the interplay effect for pencil beam scanning proton therapy of pancreatic cancer patients
}

\author{
Kai Dolde ${ }^{1,2,3^{*}+} \mathbb{D}$, Ye Zhang ${ }^{4 \dagger}$, Naved Chaudhri ${ }^{2,5}$, Christian Dávid ${ }^{3,6}$, Marc Kachelrieß ${ }^{6}$, Antony John Lomax ${ }^{4,7}$, \\ Patrick Naumann ${ }^{8}$, Nami Saito ${ }^{8}$, Damien Charles Weber ${ }^{4}$ and Asja Pfaffenberger ${ }^{1,2}$
}

\begin{abstract}
Background: Time-resolved volumetric magnetic resonance imaging (4DMRI) offers the potential to analyze 3D motion with high soft-tissue contrast without additional imaging dose. We use 4DMRI to investigate the interplay effect for pencil beam scanning (PBS) proton therapy of pancreatic cancer and to quantify the dependency of residual interplay effects on the number of treatment fractions.

Methods: Based on repeated 4DMRI datasets for nine pancreatic cancer patients, synthetic 4DCTs were generated by warping static 3DCTs with 4DMRI deformation vector fields. 4D dose calculations for scanned proton therapy were performed to quantify the interplay effect by CTV coverage (v95) and dose homogeneity (d5/d95) for incrementally up to 28 fractions. The interplay effect was further correlated to CTV motion characteristics. For quality assurance, volume and mass conservation were evaluated by Jacobian determinants and volume-density comparisons.

Results: For the underlying patient cohort with CTV motion amplitudes $<15 \mathrm{~mm}$, we observed significant correlations between CTV motion amplitudes and both the length of breathing cycles and the interplay effect. For individual fractions, tumor underdosage down to $\mathrm{v} 95=70 \%$ was observed with pronounced dose heterogeneity $(\mathrm{d} 5 / \mathrm{d} 95=1.3)$. For full $\times 28$ fractionated treatments, we observed a mitigation of the interplay effect with increasing fraction numbers. On average, after seven fractions, a CTV coverage with $95-107 \%$ of the prescribed dose was reached with sufficient dose homogeneity. For organs at risk, no significant differences were found between the static and accumulated dose plans for 28 fractions.

Conclusion: Intrafractional organ motion exhibits a large interplay effect for PBS proton therapy of pancreatic cancer. The interplay effect correlates with CTV motion, but can be mitigated efficiently by fractionation, mainly due to different breathing starting phases in fractionated treatments. For hypofractionated treatments, a further restriction of motion may be required. Repeated $4 \mathrm{DMRI}$ measurements are a viable tool for pre- and post-treatment evaluations of the interplay effect.
\end{abstract}

Keywords: Proton therapy, Pencil beam scanning, Interplay effect, 4D-MRI, 4D dose calculations, Pancreatic cancer

\footnotetext{
* Correspondence: k.dolde@dkfz.de

${ }^{\dagger}$ Kai Dolde and Ye Zhang contributed equally to this work.

${ }^{1}$ Medical Physics in Radiation Oncology, German Cancer Research Center

(DKFZ), Im Neuenheimer Feld 280, 69120 Heidelberg, Germany

${ }^{2}$ National Center for Radiation Research in Oncology (NCRO), Heidelberg

Institute for Radiooncology (HIRO), Im Neuenheimer Feld 280, 69120

Heidelberg, Germany

Full list of author information is available at the end of the article
}

C The Author(s). 2019 Open Access This article is distributed under the terms of the Creative Commons Attribution 4.0 International License (http://creativecommons.org/licenses/by/4.0/), which permits unrestricted use, distribution, and

reproduction in any medium, provided you give appropriate credit to the original author(s) and the source, provide a link to the Creative Commons license, and indicate if changes were made. The Creative Commons Public Domain Dedication waiver (http://creativecommons.org/publicdomain/zero/1.0/) applies to the data made available in this article, unless otherwise stated. 


\section{Introduction}

Pancreatic cancer is one of the leading causes of cancer deaths and shows a low 5-year survival rate of 5-20\%, depending on stage at diagnosis $[1,2]$. Currently, surgery remains the only potential possibility for curative treatment in localized disease, whereas radiotherapy (RT) and chemotherapy or combinations of both are used to improve patient survival in unresectable locally advanced stage.

Nevertheless, new RT techniques are considered more effective [3].

RT treatments of the pancreas are challenging due to the limited tolerance doses of adjacent organs at risk (OARs). While intensity-modulated radiation therapy (IMRT) is already able to decrease the doses to the GI-tract and the liver compared to 3D-conformal RT [4], particle therapy is a more promising modality to further reduce OAR toxicity by making use of the physical characteristics of the "Bragg-peak" [5]. Several encouraging preliminary results have been reported from clinical trials using carbon ions [6, 7] or proton radiotherapy with concomitant chemotherapy $[8,9]$.

However, the advantages of a high spatial accuracy in particle therapy are partly counteracted by various types of uncertainties, especially in the presence of intrafractional respiration-induced organ motion, which may lead to density changes in the proton beam paths. These changes are known to potentially cause heterogeneous dose distributions in the tumor and over- or undershooting of the target $[10,11]$.

For pencil beam scanning (PBS) particle therapy [12], the resulting interplay effects [13] between beam and tumor are considerable. Interplay effects have been investigated for different anatomical sites, such as lung [14] and liver tumors [15, 16], and various motion management techniques (e.g. rescanning, gating, breath-hold) have been proposed and analyzed [17-19] to mitigate the impact of motion. Nevertheless, compared to lung tumors whose motion can reach amplitudes of up to $53 \mathrm{~mm}$ [20], pancreatic motion is comparable to liver motion and therefore smaller due to the increased distance to the lung region [21]. However, since stomach and bowel filling influence the pancreas position in general, day-to-day pancreatic motion variations may occur.

Therefore, in this study it was the aim to evaluate the interplay effect using repeated time-resolved volumetric magnetic resonance imaging (4DMRI) for PBS proton therapy of pancreatic cancer. This approach allowed us to take into account both intra- and interfractional patient-specific organ motion and motion variations without exposing the patients to any additional imaging dose. We performed 4D dose calculations to quantify the interplay effect. The dosimetric analysis was based on synthetic 4DCTs, which were generated by warping static patient CTs by means of deformation vector fields, extracted from repeated 4DMRI data sets of patients.

Such an approach was initially developed for liver [22] and then utilized for one pancreas case in a proof-of-principle study [23]. In this previous methodical paper, we showed the feasibility of the synthetic 4DCT approach for pancreatic cancer, demonstrated the method by evaluating the statistical evolution of the interplay effect as a function of treatment fractionation and reported on the mitigation of the interplay effect by fractionation for one example pancreas case. However, due to the lack of statistics, it was not possible to quantitatively conclude on the dosimetric impact of pancreas motion, the number of required treatment fractions for an acceptable mitigation, the impact of the interplay effect on organs at risk and its correlations to tumor motion characteristics. In another recent study, which investigated the interplay effect for a cohort of 14 pancreatic cancer patients based on single 4DCTs for proton or carbon ion treatments [24], it was only possible to report the magnitude of interplay effect in a single fraction. Consequently, the efforts of the two last-mentioned studies were combined here, to evaluate 9 pancreatic cancer patients through the novel 4DMRI procedures with up to six repeated 4DMRI data sets per patient. Such repeated 4D data sets enabled a longitudinal analysis of the interplay effect as a function of the number of treatment fractions. In this study, we first aimed to quantify the patient-specificity of both day-to-day motion variations and the fractionation-induced interplay mitigation on the planned target dose and OARs. Second, we statistically determined the interplay impact on organs at risk and evaluated correlations and factors that influence the magnitude of the interplay effect. Finally, we investigated the clinical applicability of our approach with respect to a possible 4DMRI-based pre-treatment estimation of the evolution of the interplay effect along the treatment course.

\section{Material and methods}

\section{Patient data}

This study comprises a cohort of 9 pancreatic cancer patients, ( 6 females, 3 males) with a mean age of 65.9 years (50-82 years), who received either proton or carbon ion beam therapy at the Heidelberg Ion-Beam Therapy Center (HIT). Written informed consent for proton/carbon treatment and repeated MR imaging for positioning control as an individual treatment approach was obtained from all patients. For each patient, a 3D treatment planning CT, acquired under free breathing, was available on which the gross tumor volume (GTV), the clinical target volume (CTV), the internal target volume (ITV), the planning target volume (PTV) and the OARs 
were defined [25]. The GTV and CTV were delineated by a radiation oncologist, whereas the ITV was generated by means of the union of all CTVs within all breathing phases of an acquired 4DCT measurement. The PTV was defined by adding an isotropic margin of $5 \mathrm{~mm}$ around the ITV.

The 4DMRI data were acquired at a $1.5 \mathrm{~T}$ MR scanner (Magnetom Aera, Siemens Healthcare, Erlangen, Germany), using a T1-weighted gradient echo sequence with radial stack-of-stars sampling and subsequent iterative 4D-reconstruction, based on a k-space-center self-gating signal [26]. A wooden flat table top was used at the MR scanner to ensure a reproducible positioning of the patient during imaging and treatment. The individual settings and parameters for the respective patients, as well as the time between the acquired 4DMRI scans can be found in Table 1. Up to six 4DMRI scans were acquired along the respective patient treatment course per patient.

The numbers of acquired 4DMRI scans per patients differed in our cohort since some patients were treated with carbon ions within 3 weeks whereas others had proton irradiations over 5-6 weeks and thus were available for more MRI scans. In addition, MR scanning time capacities did not allow a weekly MR scan in every patient.

All patients except P8 were treated with two oblique posterior beams. P8 was treated from anterior with only one field $\left(340^{\circ}\right)$, however, to allow a better comparison with the results for the other patients, a second field with $20^{\circ}$ was included in the calculations for this patient.

For patients with less than five available 4DMRI data sets, the length of the breathing cycle of the available 4DMRI data was artificially varied between 3 and $10 \mathrm{~s}$ such as to generate different motion inputs $(\mathrm{m})$ for the subsequent $4 \mathrm{D}$ dose calculation and thereby simulate day-to-day motion variations. The respective number of motion inputs $(\mathrm{m})$ for each patient is listed in Table 2.
For the simulated breathing cycles, parentheses are added in which the respective underlying input motion is given, e.g. $3.0(\mathrm{~m} 1)$ is the 4DMRI data set of measurement 1 , where the breathing cycle was changed to be $T=3.0 \mathrm{~s}$.

\section{Synthetic 4DCT from 4DMRI}

The workflow, starting from 4DMRI data acquisition to the final 4D dose calculations for fractionated PBS proton therapy, is illustrated in Fig. 1. In short, for each patient, synthetic 4DCTs, called 4DCT(MRI) in the following, were generated by warping a $3 \mathrm{D}$ treatment planning $\mathrm{CT}$ of the respective patient with deformation vector fields (DVFs), extracted from 4DMRI data. The end-exhalation breathing phase EEX of each 4DMR image was registered to all other breathing phases $j$ of the same measurement (20 breathing phases in total) by means of deformable image registration (DIR), using the open-source software plastimatch (www.plastimatch.org) and the Demons algorithm [27]. In detail, the ITK implementation of the demons algorithm, using fast-symmetric-forces, was used with four different plastimatch stages and different scales of resolution and demons step lengths. The 3D planning CT was rigidly registered to the EEX MR image and the DVFs were used to deform the 3DCT to obtain a synthetic 4DCT(MRI) for each 4DMRI data set. The workflow is described in further detail in [23].

\section{CTV motion analysis and deformation field QA}

The CTV motion was extracted from the 4DCT(MRI) by applying a binary CTV mask to the DVFs which results in motion distributions of all voxels within the CTV delineation for each breathing phase with respect to $E E X$.

For DIR quality assurance, Jacobian determinants were calculated inside the OAR delineations (liver, kidneys, bowel) for each 4DCT(MRI), using the DVF between

Table 1 Number of available 4DMR images, patient positioning, beam angels of both fields (F0, F1), CTV volume and time between consecutive 4DMRI data sets

\begin{tabular}{|c|c|c|c|c|c|c|}
\hline \multirow[t]{2}{*}{ Patient } & \multirow[t]{2}{*}{ \# 4DMRI } & \multirow[t]{2}{*}{ Positioning } & \multicolumn{2}{|c|}{ Beam angle $\left[{ }^{\circ}\right]$} & \multirow[t]{2}{*}{ CTV volume $[\mathrm{cc}]$} & \multirow{2}{*}{$\begin{array}{l}\text { Time between consecutive } \\
\text { 4DMRI scans [days] }\end{array}$} \\
\hline & & & FO & F1 & & \\
\hline$\overline{P 1}$ & 2 & supine & 160 & 210 & 51.9 & 1 \\
\hline P2 & 6 & supine & 160 & 210 & 33.0 & $15 / 5 / 9 / 3 / 10$ \\
\hline P3 & 5 & supine & 160 & 210 & 95.1 & $6 / 9 / 5 / 11$ \\
\hline P4 & 2 & prone & 20 & 340 & 87.8 & 21 \\
\hline P5 & 1 & prone & 20 & 340 & 194.8 & - \\
\hline P6 & 1 & prone & 20 & 340 & 115.1 & - \\
\hline P7 & 1 & supine & 160 & 200 & 112.0 & - \\
\hline P8 & 3 & supine & 20 & 340 & 62.4 & $15 / 24$ \\
\hline P9 & 2 & prone & 20 & 340 & 46.0 & 7 \\
\hline
\end{tabular}


Table 2 Number of input motion patterns $m$ for the 4D dose calculation and respective breathing cycles $T$ [s] for P1-P9

\begin{tabular}{|c|c|c|c|c|c|c|c|c|c|}
\hline & P1 & P2 & P3 & P4 & P5 & P6 & P7 & P8 & P9 \\
\hline$m 1$ & 7.1 & 3.8 & 9.7 & 3.7 & 3.7 & 5.6 & 3.0 (m5) & 4.7 & 3.7 \\
\hline$m 2$ & $3.0(\mathrm{~m} 1)$ & 2.8 & 8.2 & $3.0(\mathrm{~m} 1)$ & $3.0(\mathrm{~m} 1)$ & $3.0(\mathrm{~m} 1)$ & $5.0(\mathrm{~m} 5)$ & $3.0(\mathrm{~m} 1)$ & $3.0(\mathrm{~m} 1)$ \\
\hline m3 & $5.5(\mathrm{~m} 1)$ & 3.7 & 8.8 & $5.5(\mathrm{~m} 1)$ & $5.5(\mathrm{~m} 1)$ & $5.5(\mathrm{~m} 1)$ & 7.0 (m5) & $10.0(\mathrm{~m} 1)$ & $5.5(\mathrm{~m} 1)$ \\
\hline$m 4$ & $10.0(\mathrm{~m} 1)$ & 3.7 & 8.4 & $8.0(\mathrm{~m} 1)$ & $8.0(\mathrm{~m} 1)$ & $8.0(\mathrm{~m} 1)$ & 9.0 (m5) & 5.6 & 2.9 \\
\hline$m 5$ & 4.8 & 3.7 & 6.7 & 5.9 & $10.0(\mathrm{~m} 1)$ & $10.0(\mathrm{~m} 1)$ & 10.0 & $3.0(\mathrm{~m} 4)$ & $3.0(\mathrm{~m} 4)$ \\
\hline$m 6$ & $3.0(\mathrm{~m} 5)$ & 2.8 & & 3.0 (m5) & & & & $10.0(\mathrm{~m} 4)$ & $5.5(\mathrm{~m} 4)$ \\
\hline$m 7$ & 5.5 (m5) & & & 5.5 (m5) & & & & 5.6 & \\
\hline$m 8$ & 10.0 (m5) & & & $10.0(\mathrm{~m} 5)$ & & & & $3.0(\mathrm{~m} 7)$ & \\
\hline$m 9$ & & & & & & & & $10.0(\mathrm{~m} 7)$ & \\
\hline
\end{tabular}

the EEX and end-inhalation breathing phase EIN to evaluate volume conservation.

With respect to mass conservation, the delineated volume $V_{E E X}$ of each OAR on the EEX phase of each 4DCT(MRI) was multiplied by the estimated physical density $\rho_{E E X}$ for each voxel inside the delineation, obtained from a generic HU-to-density lookup table [28], to obtain the respective mass $m_{E E X}$. Then, $m_{E I N}=V_{E I N}$. $\rho_{E I N}$ was calculated with $V_{E I N}$ being derived by warping the OAR delineation from $E E X$ to EIN using the respective DVF and the respective density information $\rho_{E I N}$. Finally, the ratio $m_{E E X} / m_{E I N}$ was considered as an estimate of mass conservation.

\section{Treatment planning and 4D dose calculation}

For all patients, PBS proton therapy plans were used with $1.8 \mathrm{~Gy}(\mathrm{RBE})$ per fraction $(\mathrm{RBE}=1.1)$ and 28 fractions, employing the beam angles of the treatments that had actually been delivered, see Table 1 .

A 3D single field uniform dose (SFUD) plan with two fields for PBS proton therapy was calculated on the 3DCT for each patient. 4D dose calculations were performed by means of the PSI 4D pencil beam proton dose calculation algorithm [22, 29], taking into account the density changes, extracted from the $4 \mathrm{DCT}(\mathrm{MRI})$, the respective breathing cycle $T$ for motion input $m$ (see Table 1) and the beam dynamics of the PSI-Gantry2 [18], e.g. the time stamps and weights of each individual pencil beam. For each field, the 4D dose distributions for 20 different initial breathing starting phases of the patient, derived from the 20 available different breathing phases of each 4DMRI data set, were calculated.

For every fraction of the simulated 28 fraction PBS proton treatments, such a $4 \mathrm{D}$ dose distribution was calculated by random selection of both the underlying

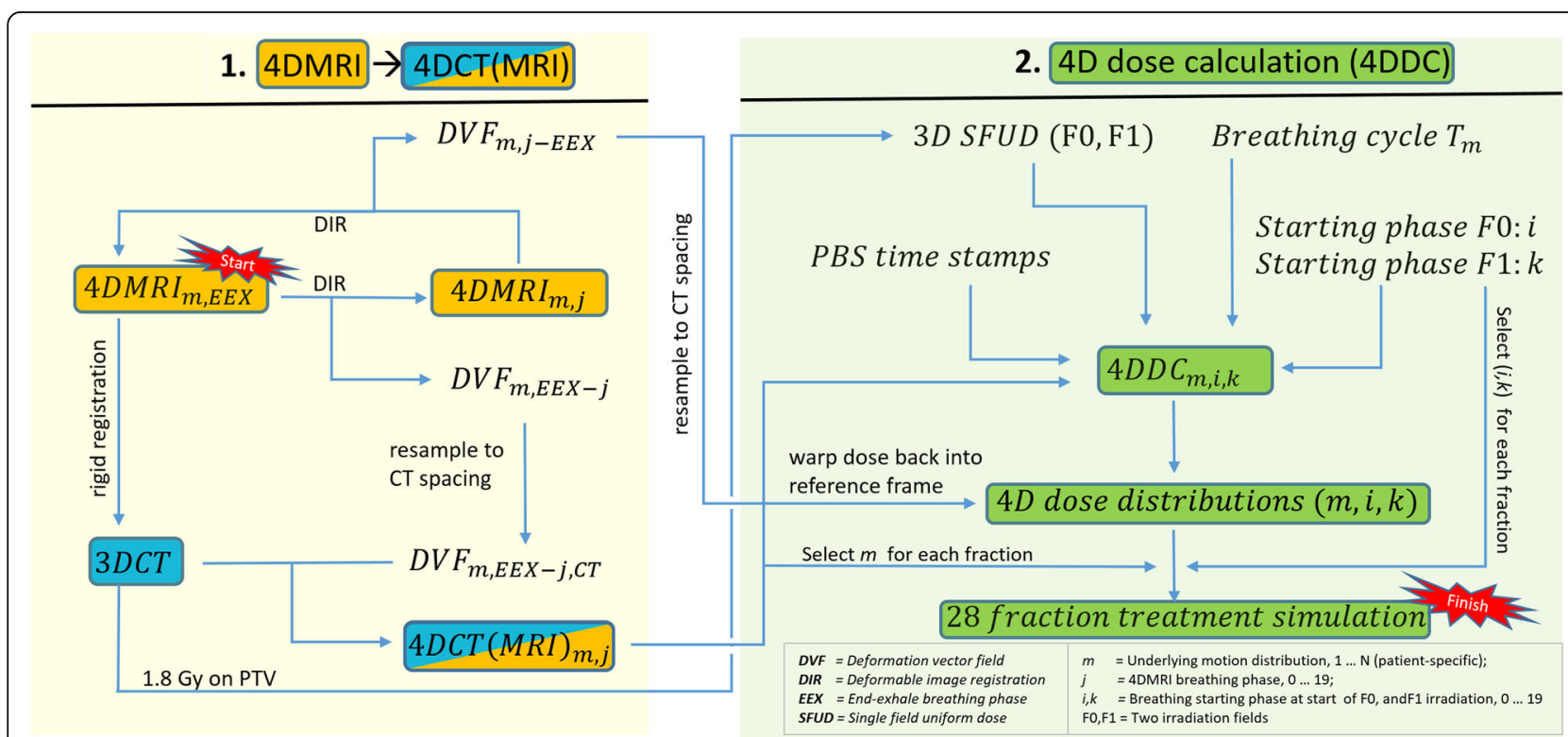

Fig. 1 Schematic Workflow from 4DMRI via synthetic 4DCT(MRI) to 4D dose calculations for fractionated PBS proton therapy of pancreatic cancer 
4DCT $(\mathrm{MRI})_{m}$ density information and the breathing starting phases $(i, k)$ for both fields F0 and F1, see Fig. 1.

The resulting interplay effect was analyzed with respect to dose homogeneity in the CTV $(d 5 / d 95)$, CTV coverage $(v 95, v 107)$, mean dose $d_{\text {mean }}$ and near maximum dose $d 2$ in the CTV for the 3D dose calculation (3DDC), as well as for the 4D dose distributions for single fractions (4Dx1) and 28 fractions (4Dx28), respectively. Here, $d 5, d 95$ and $d 2$ denote the relative doses that 5,95 and $2 \%$ of the volume receive, respectively, and $v 95$ and $v 107$ are the percentage volumes, that receive at least 95 and $107 \%$ of the prescribed dose.

Moreover, the dose-volume-histograms (DVHs) for OARs, namely left/right kidney, spinal cord, liver and bowel were analyzed with respect to $d_{\text {mean }}, d 2$ and $v 30$, where $v 30$ denotes the volume that receives at least $30 \%$ of the dose. The results were tested for significance by means of a single-sided Wilcoxon rank-sum test with a confidence level of $95 \%(\alpha=0.05)$.

\section{Correlation analysis}

Inter-patient correlations between CTV motion amplitudes, length of breathing cycles and the interplay effect, assessed as $\mathrm{d} 5 / \mathrm{d} 95$ of a single fraction, were determined by calculations of weighted correlations coefficients $\rho_{w}$. The respective numbers of repeated 4DMRI data sets were used as weights for each patient, in compliance with previous publications [30, 31].

The statistical significance was calculated assuming a $\mathrm{t}$-distribution of the data for small sample sizes with $N=9$. In this case, the test statistic $t=\rho_{w} \cdot \sqrt{(N-2) /\left(1-\rho_{w}^{2}\right)}$ was calculated and compared to the tabulated critical value $t_{\text {crit }}=1.833$ of the $\mathrm{t}$-distribution for a one-sided test with a confidence level of $95 \%$. Statistical significance was determined for $t>t_{\text {crit }}$.

Furthermore, the correlation coefficient $\rho_{m}$ between CTV volumes, which were considered constant for all fractions, and the mean interplay effects were calculated. The dependency of patient positioning (supine/prone) and beam angles on the interplay effect was analyzed by comparison of mean d5/d95 values. Additionally, for patients with multiple available 4DMRI data sets, intra-patient dependencies between CTV motion amplitudes, length of breathing cycles and the interplay effect (d5/d95) were analyzed.

\section{Longitudinal interplay investigation for a possible clinical application}

In a possible clinical scenario, pre-treatment acquisitions of 4DMRI data sets could be used to estimate the statistical evolution of the interplay effect during fractionation. We investigated the uncertainty of such an estimation based on a single pre-treatment 4DMRI as opposed to multiple 4DMRI data sets acquired over the treatment course by comparing the respective evolution of the interplay effect, assessed as $\mathrm{d} 5 / \mathrm{d} 95$. For all patients with $\geq 2$ available 4DMRI scans (P1, P2, P3, P4, P8, P9), we performed 30 simulations each of the entire $\times 28$ fractionated treatment scheme, taking into account only the first motion pattern (m1) to simulate a single pre-treatment 4DMRI acquisition, or all motion patterns, respectively. For the latter, for each treatment fraction the respective motion pattern was randomly sampled from the available patterns for each patient.

\section{Results}

Motion extraction from 4DMRI and vector field QA

The extracted CTV motion distributions in inferior-superior (IS), anterior-posterior (AP) and left-right (LR) direction are illustrated in Fig. 2 for all patients. They show the patient-specificity of the motion patterns as well as interfractional changes in CTV motion, extracted from the 4DCT(MRI). For this patient cohort, maximum CTV motion amplitudes of $15 \mathrm{~mm}$ were present with comparably small mean values of $\leq 8 \mathrm{~mm}$ in IS and $\leq 3 \mathrm{~mm}$ in AP and LR direction, respectively. Maximum intra-patient day-to-day differences in mean and maximum motion amplitudes of up to $5.3 \mathrm{~mm}$ and $8.1 \mathrm{~mm}$ (for P8), respectively, arose in IS direction, the dominant direction of motion.

The evaluation of volume and mass conservation yielded a mean Jacobian determinant among all patients of $1.00 \pm 0.04$, indicating volume-conserving DIR with low regional volume changes in the OARs. A mean mass conservation coefficient value of $m_{E E X} / m_{I N}=1.07 \pm 0.05$ was derived.

\section{Static treatment plans}

The 3D SFUD plans revealed an average mean dose of $d_{\text {mean }}=99.6 \%$ (range 99.5-99.9\%) with full CTV coverage $(v 95=100 \%, v 107=0 \%)$, high dose homogeneity with $d 5 / d 95 \leq 1.03$ (range 1.01-1.03) and near maximum dose values of $d 2<103 \%$ (range 100.3-103.0\%) for 8 out of the 9 patients. For P8, slightly inferior values $\left(d_{\text {mean }}=101.4 \%, \mathrm{v} 95=100 \%, \mathrm{v} 107=6.5 \%, \mathrm{~d} 5 / \mathrm{d} 95=1.11\right.$, d2 $=108.9 \%$ ) were obtained, as illustrated in Fig. 3a, c, d.

\section{D dose distributions for single fraction}

By means of 4D dose calculations of a single fraction (4Dx1), the interplay effect of all combinations of initial breathing starting phases and underlying motion patterns was quantified in terms of $d 5 / d 95$ and $v 95$. Depending on the patient, mean $d 5 / d 95=1.05-1.14$ were obtained with maximum values of up to 1.3 . CTV coverage $v 95$ mean values of $92-100 \%$ were observed with minimum values of $70 \%$, implying pronounced 

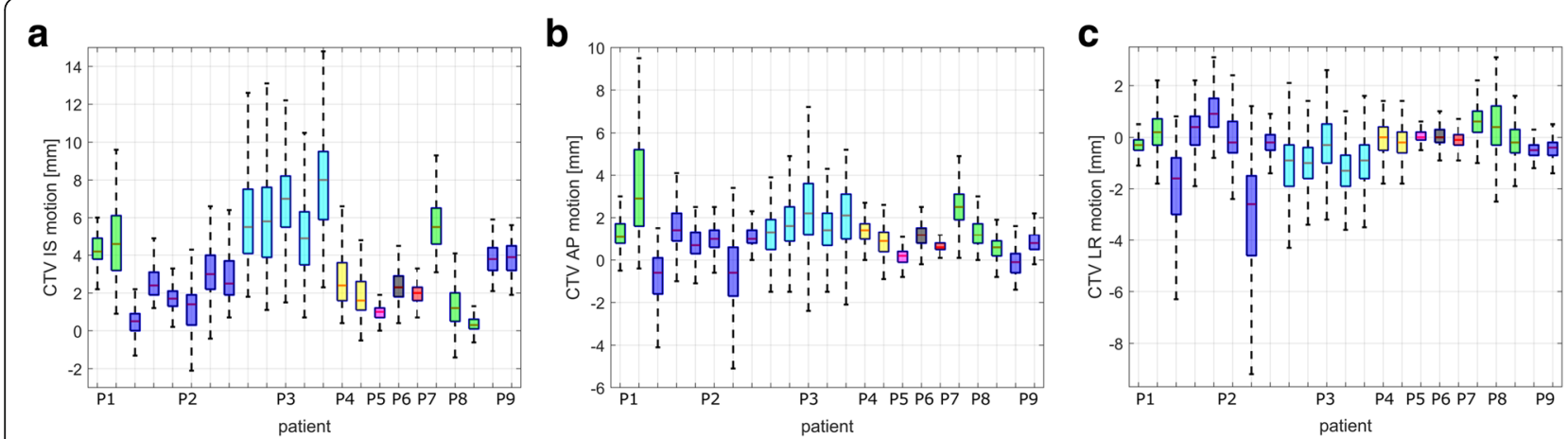

Fig. 2 Motion distributions of all voxels inside the CTV delineation between end-inhale and end-exhale breathing phases for P1-P9 in a IS, b AP and $\mathbf{c}$ LR direction for the respective numbers of available 4DMRI data sets. The whiskers indicate the $95 \%$ range of the boxplots

underdosage of the CTV. Moreover, near maximum doses $d 2$ of up to $110 \%$ were present within the CTV. These values show a strong dependency on the underlying motion input, which is discussed in detail in "Correlation analyses" section below. The calculated 3D SFUD plans, exemplary single fraction 4D dose distributions and single fraction interplay effects in terms of $d 5$ / $d 95$ and $v 95$ are displayed in Fig. 3. Pronounced hot and cold spots are visible in the 4D distributions.

\section{D dose distributions for 28 fractions treatment}

The simulated treatments with 28 fractions (4Dx28) show a patient-specific mitigation of the interplay effect as a function of the number of treatment fractions. Figure 4 displays example $d 5 / d 95$ and $v 95$ values for patients P1/P2/P6, who showed large/medium/small interplay effects in the single fraction calculations, respectively. Mean $d 5 / d 95$ decreased from 1.13/1.09/1.05 to $1.03 / 1.03 / 1.02$ for these patients after 28 fractions, mean $v 95$ reaches $100 \%$ after $11 / 6 / 2$ fractions, respectively.

We observed the same trend of an increased averaging effect of the interplay effect as a consequence of an increased number of fractions for all patients in this study. After a standard fractionation scheme with 28 fractions, the dose homogeneity $d 5 / d 95$ in the CTV was similar for both 4D and 3D dose distributions and $v 95=100 \%$ was obtained for all patients after 2-14 fractions (mean: 6.7 fractions). Moreover, $d 2$ as well as $v 107$ were reduced by fractionation. Except for P8, $v 107=0 \%$ was obtained after at most 10 fractions (mean: 5.6 fractions). For P8, $v 107=6 \%$ was observed after 28 fractions.

On average, $d_{\text {mean }}$ increased by $0.47 \% / 0.49 \%$ in the 4DDC for 1 or 28 fraction scenarios, respectively, compared to 3DDC. For $d 5 / d 95, d 2$ and $v 107$, we observed significantly higher values for 4Dx1 compared to both, 3DDC and 4Dx28, whilst $v 95$ was significantly lower for 4Dx1 compared to both 3DDC and 4Dx28, as apparent in Fig. 5.
With respect to $\mathrm{d}_{\text {mean }}$ and $d 5 / d 95$, statistically significant differences between 3DDC and 4Dx28 were observed, which, however, appear to be clinically less relevant due to the small absolute differences (mean $\mathrm{d}_{\text {mean }}=99.8 \% / 100.3 \%$ and mean $d 5 / d 95=1.028 / 1.034$ for $3 \mathrm{DDC} / 4 \mathrm{Dx} 28$, respectively). These results indicate that statistically for a large number of fractions, the interplay effect reduces to an acceptable CTV dose distribution in PBS proton therapy of pancreatic cancer.

\section{Dose to OARs}

From the DVHs for the left/right kidney, spinal cord, liver and bowel, the dose quantities $d_{\text {mean }}, d 2$ and $v 30$ were compared among 3DDC, 4Dx1 and 4Dx28. The deviations in $d_{\text {mean }}$ were $<0.5 \%$ for all considered organs at risk. With respect to mean $v 30$, deviations of $<1.2 \%$ were observed between the three different scenarios. The mean near maximum dose $d 2$ increased by $0.6-$ $3.2 \%$ for all OARs (except in the bowel, where $\mathrm{d} 2$ decreased by $1.5-2.1 \%$ ) when comparing 3DDC with both 4Dx1 and 4Dx28. Overall, no significant differences were found for the OARs, comparing the three different scenarios, see Fig. 6.

\section{Correlation analyses}

The analysis of inter-patient correlations showed significant $\left(t>t_{\text {crit }}=1.833\right)$ positive correlations between CTV motion amplitudes on the one hand, and the length of breathing cycles and the interplay effects assessed in terms of $\mathrm{d} 5 / \mathrm{d} 95$ on the other hand, as illustrated in Fig. 7. The strongest correlation was revealed between the CTV motion amplitudes and $d 5 / d 95\left(\rho_{w}=0.86, t=\right.$ 4.46). Smaller, but still significant correlations were found between breathing cycle and CTV motion amplitude $\left(\rho_{w}=0.65, t=2.26\right)$. The correlations between the length of breathing cycles and $\mathrm{d} 5 / \mathrm{d} 95\left(\rho_{w}=0.48, t=1.45\right)$ and between CTV volume and $\mathrm{d} 5 / \mathrm{d} 95\left(\rho_{m}=-0.49\right.$, $t=1.49)$ were determined to be not significant. 


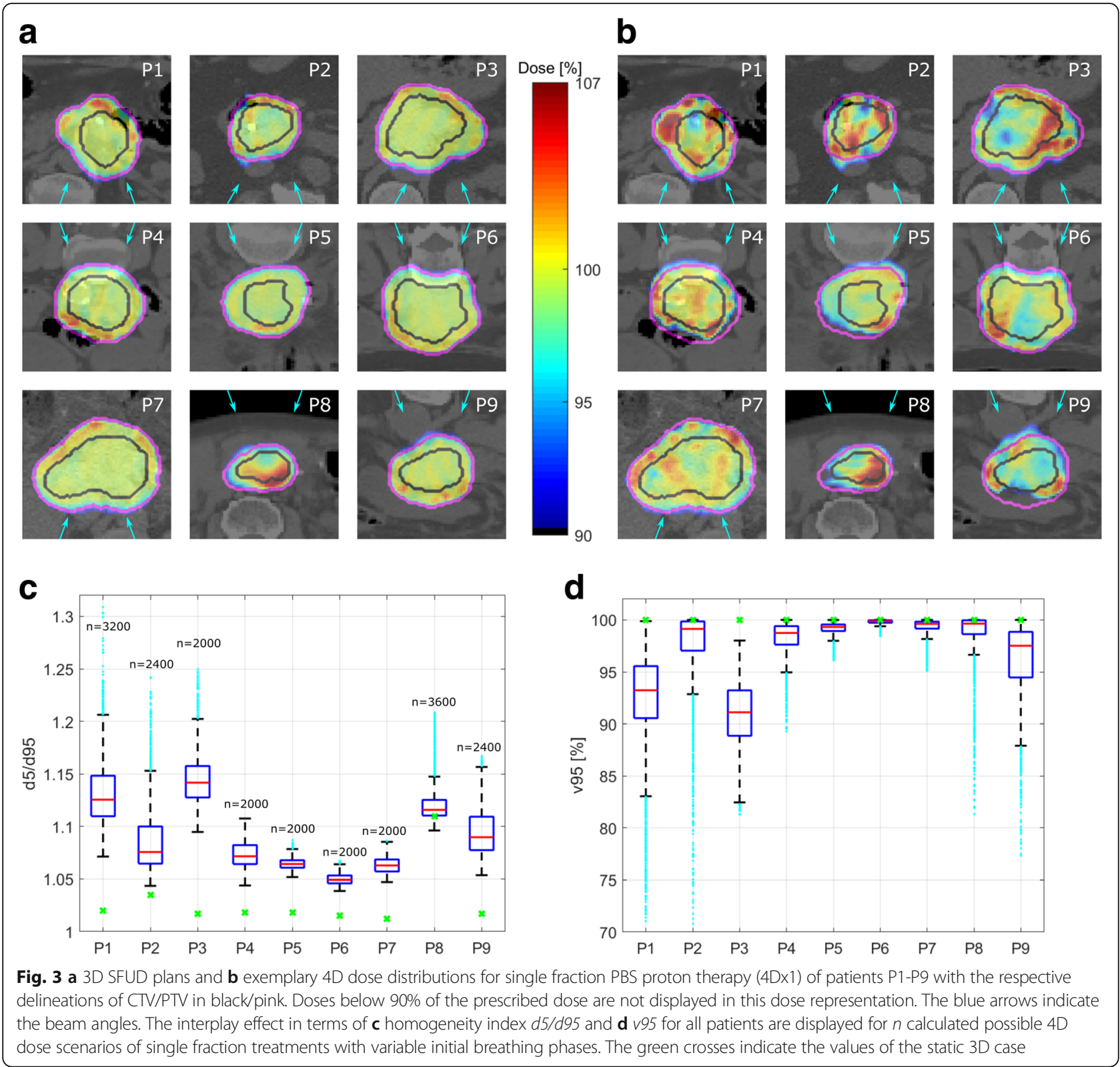

Patients in supine positioning (mean $\mathrm{d} 5 / \mathrm{d} 95=1.10$ ) showed a similar magnitude of interplay effect as patients in prone positioning (mean d5/d95 $=1.08$ ).

The intra-patient analysis revealed patient-specific both proportional and anti-proportional dependencies between CTV motion amplitude, interplay effect and length of breathing cycles with no clear tendency. Due to the small sample sizes, no correlation coefficients were determined.

\section{Longitudinal interplay investigation towards a possible clinical application}

For a pre-treatment estimation of the statistical evolution of the interplay effect during a $\times 28$ fractionated treatment scheme, we found that a single 4DMRI scan may be sufficient for patients with low day-to-day motion variations, which was the case in this study (day-to-day variations of mean motion amplitudes $\leq 5.3 \mathrm{~mm}$ ). Due to the interplay mitigation effect by variable initial breathing phases in different fractions, after 28 fractions the mean $\mathrm{d} 5 / \mathrm{d} 95$ value in 4D simulations with a single underlying 4DMRI or all available 4DMRI scans for the respective patients differed only by $0.1 \%$. For smaller numbers of fractions $(<5)$, the estimation of the interplay effect by the single-4DMRI approach differed by up to $3 \%$ compared to the multiple-4DMRI approach. Figure 8 illustrates both scenarios for P2 and P8. 

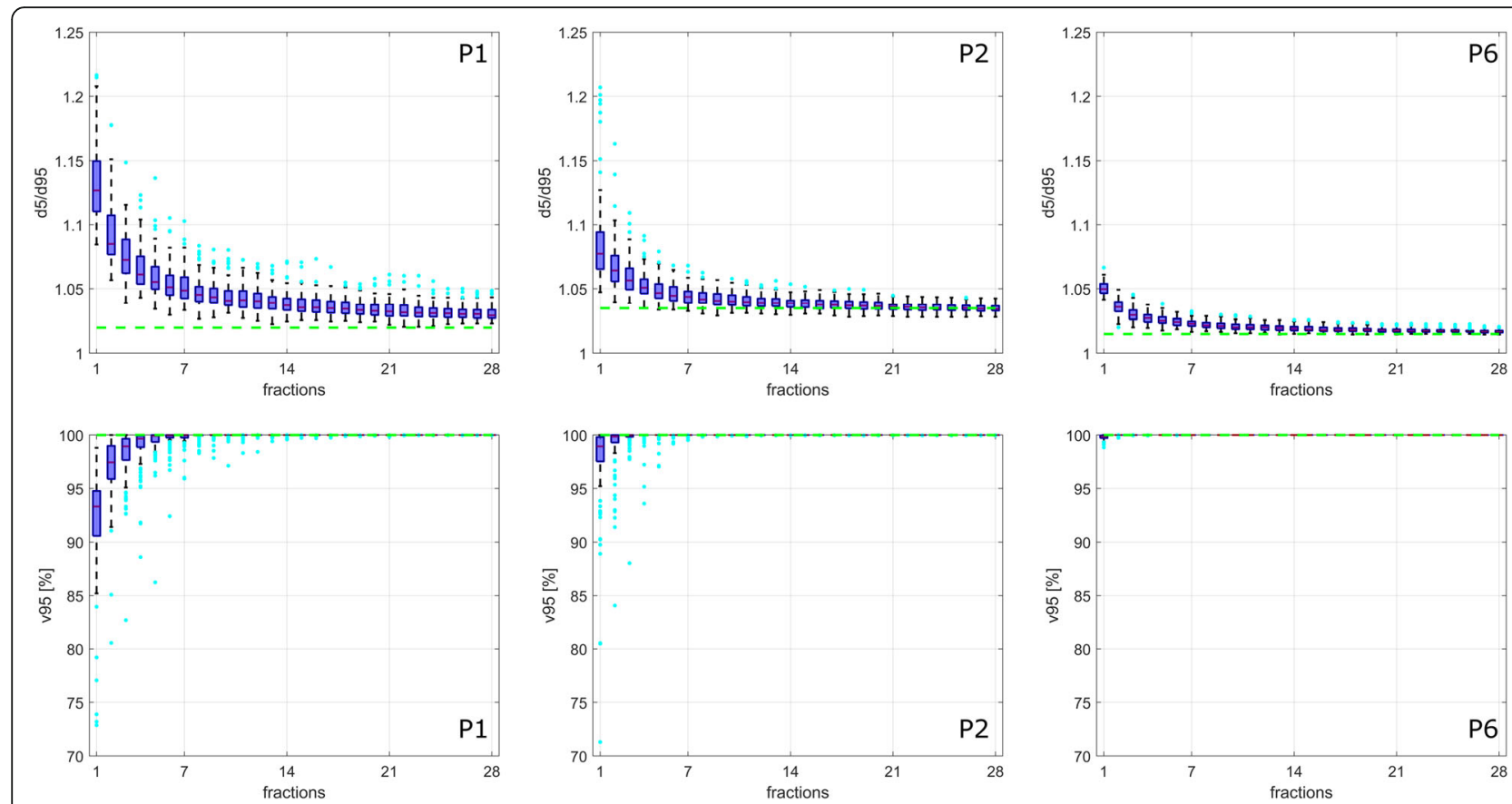

Fig. 4 Exemplary d5/d95 (a-c) and v95 (d-f) distributions for 1-28 fractions PBS proton therapy of pancreatic cancer for P1/P2/P6, who showed a large/medium/small interplay effect. The green lines indicate the static 3D case
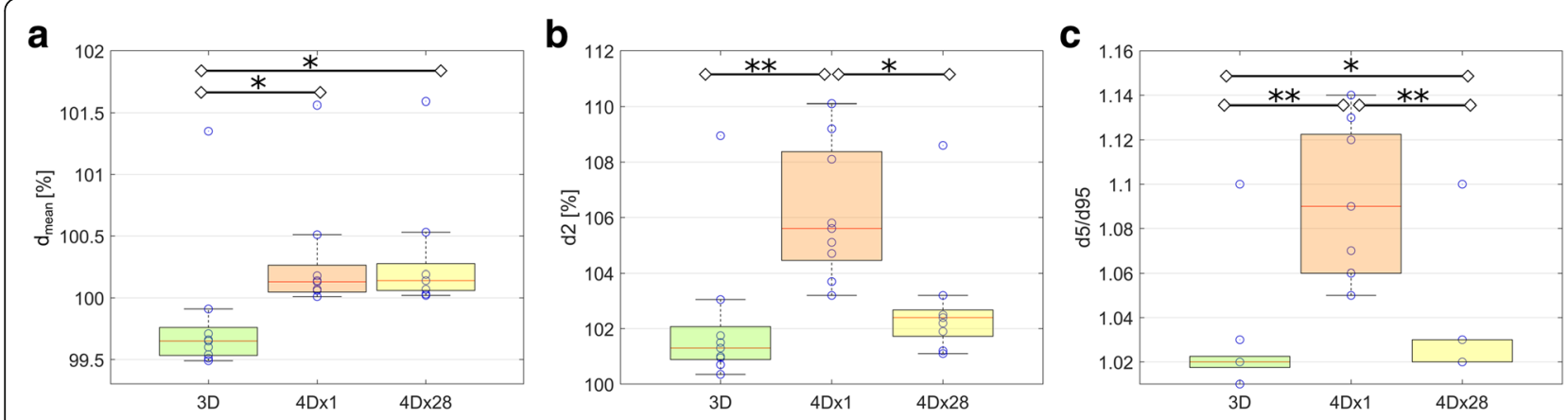

d

e
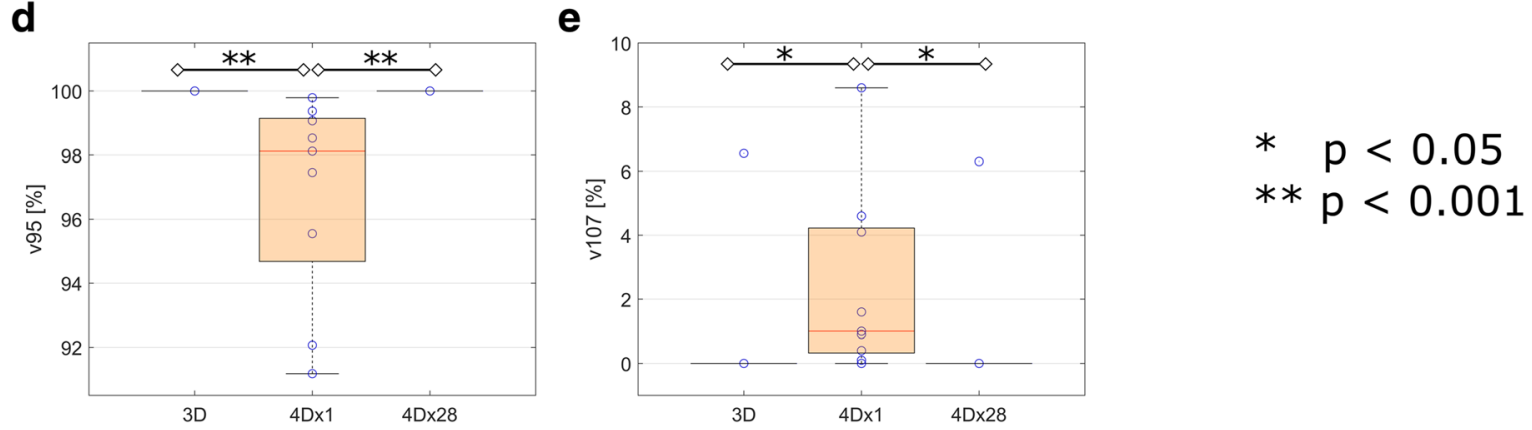

Fig. 5 CTV dose quantities $\left(d_{\text {mean }}, d 2, d 5 / d 95, v 95, v 107\right)$ for P1-P9, resulting from 3D dose calculations (3DDC), as well as from 4D dose distributions for single (4Dx1) and 28 fractions (4Dx28), respectively 

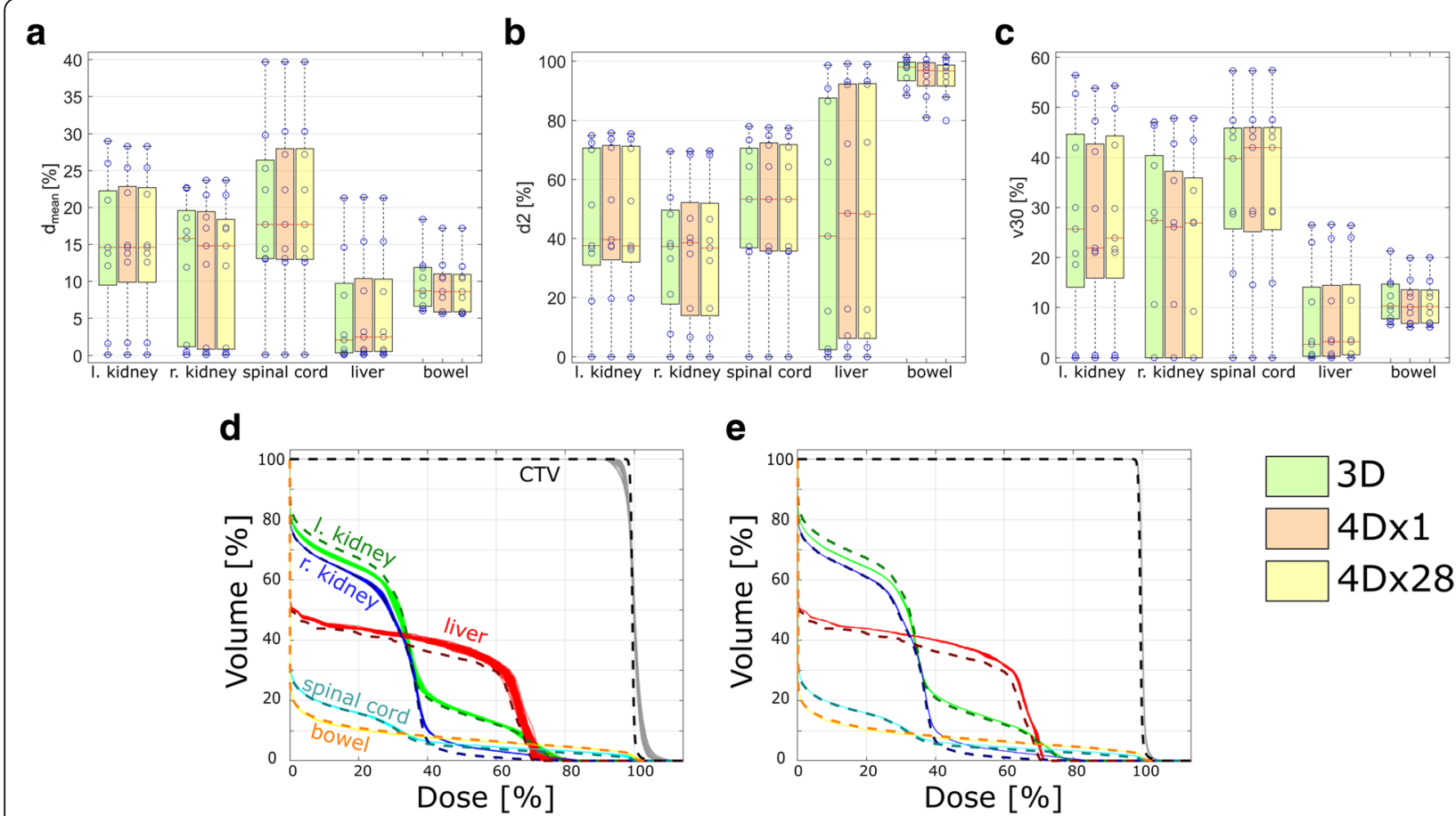

Fig. 6 The OAR dose quantities $\mathbf{a} d_{\text {mean, }} \mathbf{b} d 2$ and $\mathbf{c} v 30$ show no significant differences when comparing static dose calculations (3DDC) with 4D scenarios with single (4Dx1) and 28 fractions (4Dx28). Exemplary DVHs of P5 for $\mathbf{d} 4 \mathrm{Dx} 1$ and $\mathbf{e} 4 \mathrm{D} \times 28$ include both OARs and the CTV, as well as the respective dosimetric results from the $3 \mathrm{D}$ dose calculations in dashed lines

\section{Discussion}

This study shows a pronounced averaging effect of the interplay effect by means of fractionation for a cohort of 9 pancreatic cancer patients. On average, after 7 fractions (range 2-14), a sufficient CTV coverage of 95-107\% was obtained. This result is especially interesting with respect to hypofractionated treatments, as for instance performed in a Japanese carbon-ion dose escalation study with 8 fractions, which showed nevertheless promising outcomes [32]. However, although fractionation leads to a mitigation of the interplay effect, still in every single fraction hot and cold spots occur, whose clinical impact should be evaluated separately. For hypofractionated treatments or large motion amplitudes, additional motion mitigation could for instance be achieved by means of abdominal corsets [33, 34]. Alternatively, the choice of suitable small gating windows could further improve the resulting dose distributions and is of interest for further studies.

With respect to correlations, we found strong and medium positive inter-patient correlations between the CTV motion amplitudes on the one side and both the length of breathing cycles and the interplay effect on the other side, which is in good agreement with Dowdell et al., who found similar correlation for the interplay effect in lung proton treatments [35]. We are aware that our patient cohort shows comparably low CTV motion amplitudes, whereas pancreas motion amplitudes of $>$ $30 \mathrm{~mm}$ have been reported [36]. From the observed correlations between CTV motion amplitudes and the amount of interplay effect, we therefore expect a stronger impact of the interplay effect for large-motion patients than for our patient cohort.

We need to acknowledge several limitation in our study here. First, deformable dose mapping is generally limited by uncertainties resulting from the performed image registrations and the resulting VFs $[37,38]$. The VF-QA, performed in this study, showed sufficient Jacobian determinant values of $1.00 \pm 0.04$. In terms of mass conservation, it has been pointed out, that deformable image registrations in general may show certain uncertainties concerning mass conservation, since CT Hounsfield Units may be averaged during transformation [39]. We observed relative mass differences in the considered OARs between the EIN and EEX images of $7 \pm 5 \%$. Similar mean deviations of $5 \pm 7 \%$ have been reported for DIR of thoracic CTs with deviations up to $50 \%$ [40], which illustrate the intrinsic uncertainties of deformable image registration.

Second, the demonstrated method of warping a static CT by means of various 4DMRI vector fields holds further intrinsic limitations. Geometrical differences in the patient anatomy between different days, such as 


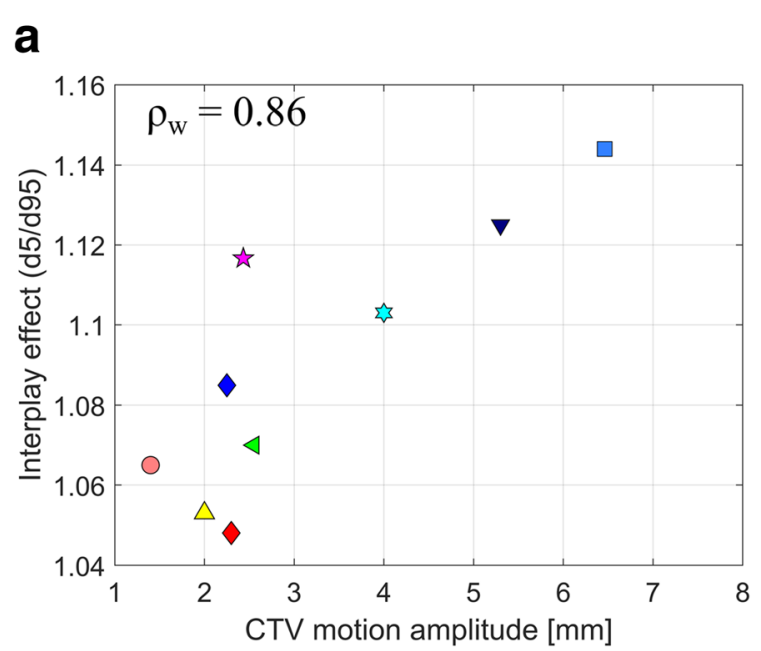

b
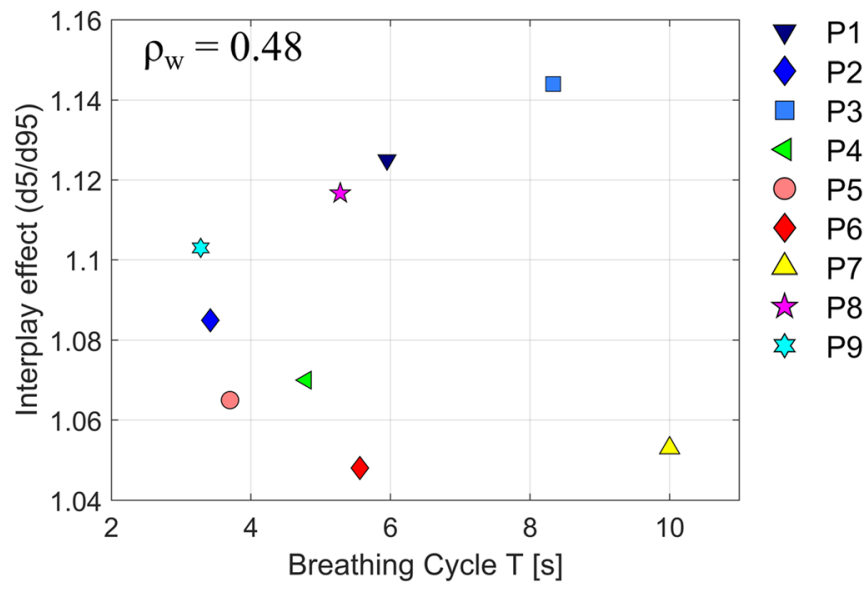

C

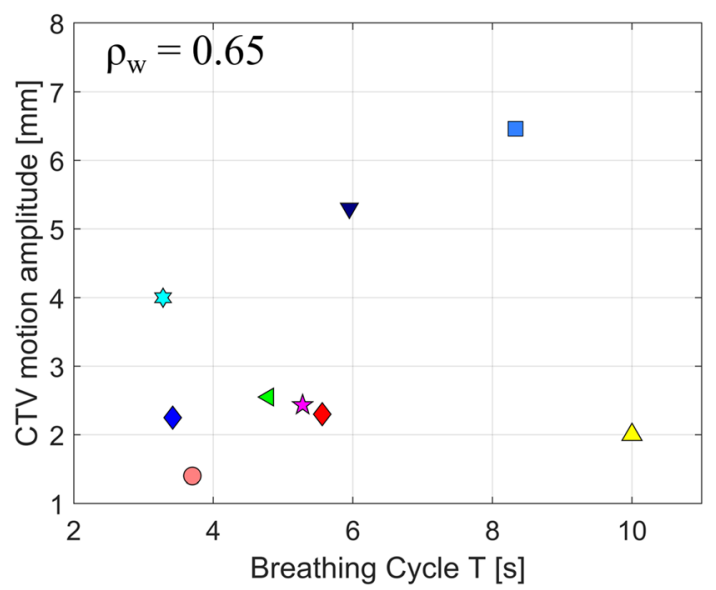

d

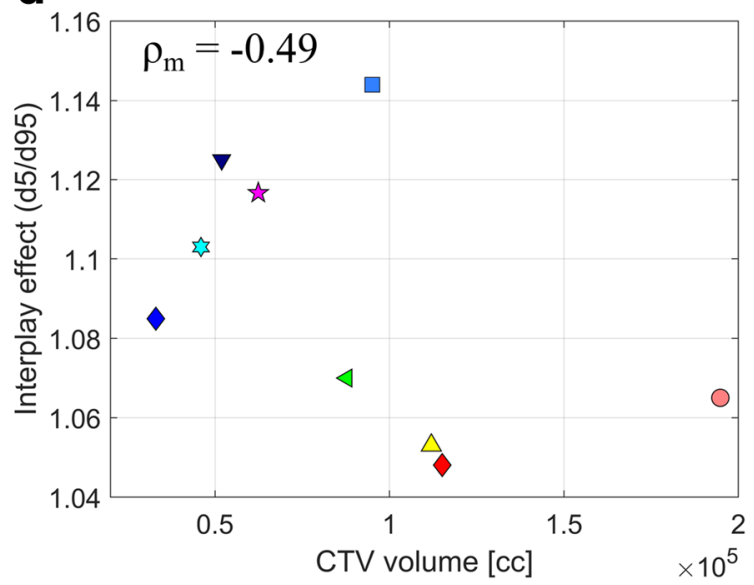

Fig. 7 Inter-patient correlations between CTV motion amplitudes, length of breathing cycles and the interplay effect assessed as d5/d95 (a-c). For each patient, the mean values of the respective quantities are displayed. The correlation between the interplay effect and the CTV volume is illustrated in (d)

translations of pancreas due to gastrointestinal distention [41], are not fully considered in this 4D treatment planning approach, as the same patient CT is deformed for all of the various motion inputs of the same patient. Moreover, the free-breathing treatment planning CTs were registered to the EEX phase of the respective 4DMR images, which visually showed a better agreement to the CT than the mid-ventilation MR images. However, a detailed statistical analysis to determine the most suitable of all available 20 breathing phases of each 4DMRI could further reduce the registration uncertainties. Alternatively, a more challenging deformable multi-modal CT-MR deformable image registration could be considered in future studies.

Furthermore, only regular breathing motion was investigated. The impact of irregular motion pattern on the interplay mitigation should be investigated in future studies. For the simulated $4 \mathrm{D}$ data sets we did not change the motion amplitudes when varying the length of breathing cycles due to the unclear intra-patient correlations between CTV motion amplitudes and length of breathing cycles. However, it is unclear whether the amplitudes would indeed be the same for different lengths of breathing cycles, which is a limitation of this study.

Finally, we would like to rephrase the importance and advantages of using repeated 4DMRI data sets of patients for subsequent $4 \mathrm{DCT}(\mathrm{MRI})$ generation and $4 \mathrm{D}$ dose calculation, compared to the conventional 4DCT approach. Being an imaging modality without any imaging dose to the patient, regular 4DMRI data could be acquired from the patients, saving $20-200$ mGy compared to a single 4DCT measurement [42]. By means of 4DMRI data, the duration of data acquisition may be extended over multiple breathing cycles, which provides more information than a single 4DCT snapshot of a single breathing cycle. 


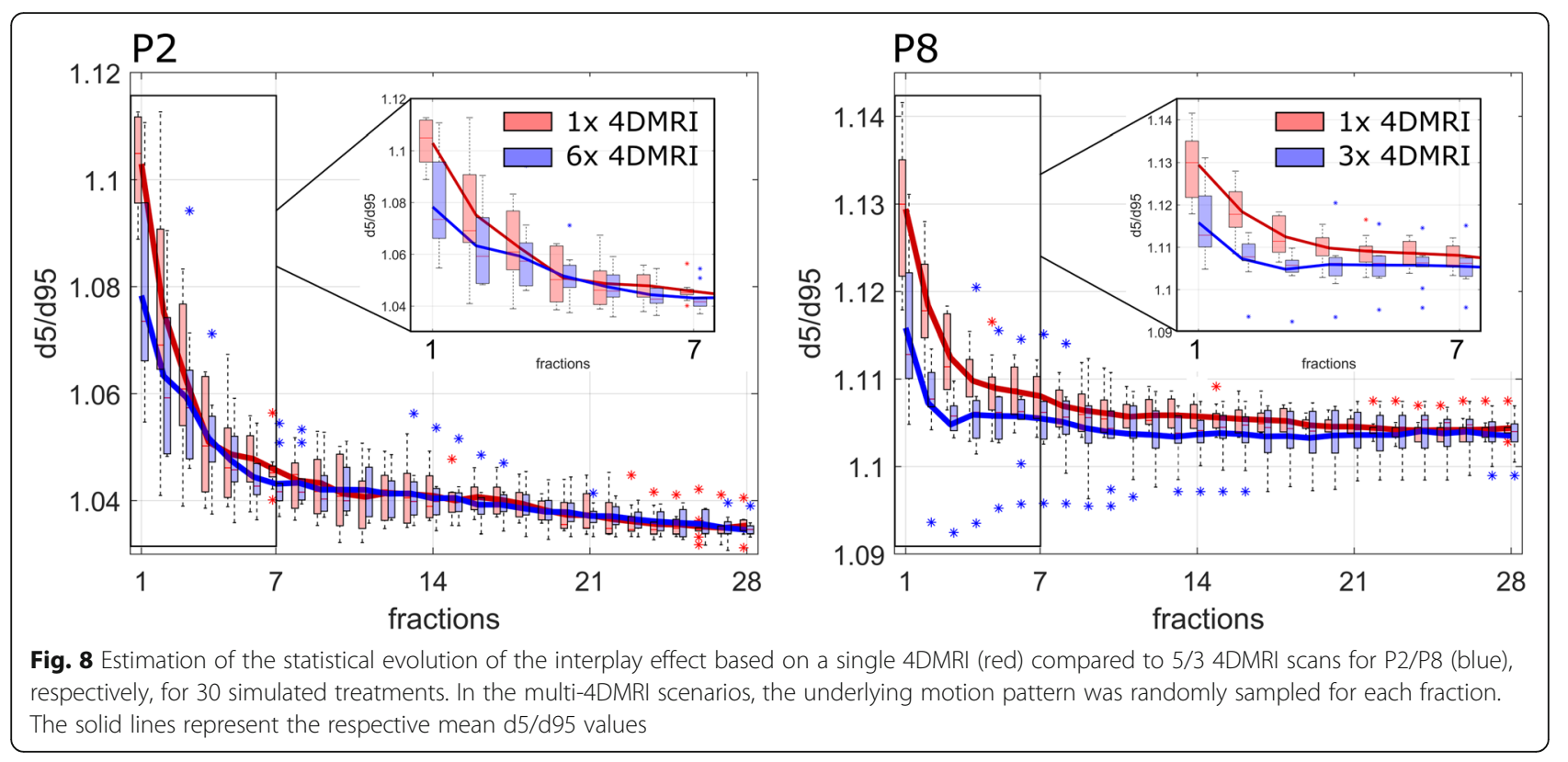

Previous studies have shown that pancreas motion shows huge day-to-day variations and one single 4DCT may not be representative for the tumor motion during a treatment [43], lasting over several weeks. Repeated 4DMRI measurements, acquired prior to the treatment, can help to observe these motion variations and could potentially be used for robust analyses or worst-case optimizations [44], based on real patient-specific motion patterns. In future scenarios, if online 4DMRI is available during irradiation on hybrid MR-Linac devices [45-48] or possible MR-proton devices, such an approach can also be used for online 4DMRI-based dose recalculations. For instance, static MR images could be acquired prior to each fraction to account for geometrical differences and patient setup errors. Then, 4DMRI could be acquired simultaneously during each irradiation fraction and the derived vector fields could be used to retrospectively calculate a $4 \mathrm{D}$ dose distribution of the day by means of the proposed method in this study and to accumulate all 4D fraction doses.

Even without online MR image-guidance, we found that our proposed method to quantify the interplay effect as a function of fractionation provides a viable tool for pre-treatment estimations of the statistical evolution of the interplay effect within an uncertainty range of a few percent for patients with low day-to-day motion variations. This is due to the fact, that variable initial breathing phases during irradiation fractions have a larger impact on the mitigation effect than small differences in breathing motion patterns. Therefore, such an approach allows an estimation of the statistical mitigation of the interplay effect by means of pre-treatment 4DMRI acquisitions. Based on such pre-treatment
4DMRI data, criteria may be defined to determine, whether additional motion limitation by abdominal compression or the application of gating criteria may be required for a specific patient.

\section{Conclusion}

We found 4D dose evaluation, based on repeated 4DMRI data sets, to be a promising methodology to investigate the mitigation of the interplay effect in PBS proton therapy of pancreatic cancer treatments. 4D investigations of a patient cohort of 9 pancreatic cancer patients with CTV motion amplitudes $<15 \mathrm{~mm}$ showed significant positive correlations between CTV motion amplitudes one the one hand and both the interplay effect and the length of breathing cycles on the other hand. We observed a gradually increased mitigation of the interplay effect with an increased number of fractions. After an average of 7 fractions, a sufficient CTV coverage of $95-107 \%$ was observed and the dose homogeneity within the CTV was similar in 3D and 4D dose distributions. For hypofractionated treatments, the study indicates a more pronounced impact of the interplay effect for pancreatic cancer treatments. For patients with low day-to-day motion variations, such as those enrolled in this study, the statistical evolution of the interplay effect along the treatment course could be estimated based on a single 4DMRI.

\section{Abbreviations}

3D: Volumetric; 3DDC: 3D dose calculation; 4D: Time-resolved volumetric; 4DCT(MRI): Synthetic 4DCT, generated from 4DMRI; 4DDC: 4D dose

calculation; 4Dx1: 4D dose distribution for a single fraction; 4Dx28: 4D dose distribution for 28 fractions; AP: Anterior-posterior; CT: Computed

tomography; CTV: Clinical target volume; d2, d5, d95: Relative dose, that 2\%/ $5 \% / 95 \%$ of the volume receive; DIR: Deformable image registration;

DVF: Deformation vector field; DVH: Dose-volume-histogram; EEX: End- 
exhalation breathing phase; EIN: End-inhalation breathing phase; GTV: Gross tumor volume; IMRT: Intensity-modulated radiation therapy; IS: Inferiorsuperior; ITV: Internal target volume; MRI: Magnetic resonance imaging; OAR: Organ at risk; PBS: Pencil beam scanning; PTV: Planning target volume; QA: Quality assurance; RT: Radiation therapy; SFUD: Single field uniform dose; v30, v95, v107: Volume, that receives 30\%/95\%/107\% of the prescribed dose

\section{Acknowledgements}

The authors would like to thank Joao Seco for his support and fruitful discussions,

\section{Funding}

The study was supported by the Helmholtz International Graduate School for Cancer Research (Heidelberg/Germany) and the "Verein zur Förderung der Tumortherapie mit schweren Ionen e.V." (Darmstadt/Germany).

\section{Availability of data and materials}

The datasets generated and analyzed during the current study are available from the corresponding author on reasonable request.

\section{Authors' contributions}

AP initiated the 4DMRI study for pancreatic cancer motion investigation. PN recruited the patients for this pilot study. CD and MK provided both software and support for the $4 \mathrm{DMRI}$ reconstructions. NC provided important information on the treatment plans. KD and $Y Z$ generated the synthetic $4 D C T$ s and performed the $4 D$ dose calculations. AP, DCW, AJL and NS contributed with fruitful discussions, coordination and, as well as PN, with critical revisions of the manuscript. KD performed the statistical analyses and drafted the manuscript. All authors read and approved the final manuscript.

\section{Ethics approval and consent to participate}

Written informed consent for proton/carbon treatment and repeated MR imaging for positioning control as an individual treatment approach was obtained from all patients.

\section{Consent for publication}

Written informed consent for permission to publish treatment related data in an anonymized way was obtained from all patients.

\section{Competing interests}

The authors declare that they have no competing interests.

\section{Publisher's Note}

Springer Nature remains neutral with regard to jurisdictional claims in published maps and institutional affiliations.

\section{Author details}

${ }^{1}$ Medical Physics in Radiation Oncology, German Cancer Research Center (DKFZ), Im Neuenheimer Feld 280, 69120 Heidelberg, Germany. ${ }^{2}$ National Center for Radiation Research in Oncology (NCRO), Heidelberg Institute for Radiooncology (HIRO), Im Neuenheimer Feld 280, 69120 Heidelberg, Germany. ${ }^{3}$ Department of Physics and Astronomy, Heidelberg University, Im Neuenheimer Feld 226, 69120 Heidelberg, Germany. ${ }^{4}$ Center for Proton Therapy, Paul Scherrer Institute (PSI), 5232 Villigen-PSI, Switzerland. ${ }^{5}$ Heidelberg lon-Beam Therapy Center (HIT), Im Neuenheimer Feld 450, 69120 Heidelberg, Germany. ${ }^{6}$ X-Ray Imaging and Computed Tomography, German Cancer Research Center (DKFZ), Heidelberg, Germany. ${ }^{7}$ Department of Physics, ETH Zurich, 8092 Zurich, Switzerland. ${ }^{8}$ Department of Radiation Oncology, University Clinic Heidelberg, Im Neuenheimer Feld 672, 69120 Heidelberg, Germany.

Received: 11 October 2018 Accepted: 24 January 2019

Published online: 07 February 2019

\section{References}

1. Siegel RL, Miller KD, Jemal A. Cancer statistics, 2015. CA Cancer J Clin. 2015;65(1):5-29.

2. Raimondi S, Maisonneuve P, Lowenfels AB. Epidemiology of pancreatic cancer: an overview. Nat Rev Gastroenterol Hepatol. 2009;6(12):699-709.

3. Hazard L. The role of radiation therapy in pancreas Cancer. Gastrointest Cancer Res. 2009;3(1):20-8.
4. van der Geld YG, van Triest B, Verbakel WF, van Sörnsen de Koste JR, Senan S, et al. Evaluation of four-dimensional computed tomographybased intensity-modulated and respiratory-gated radiotherapy techniques for pancreatic carcinoma. Int J Radiat Oncol Biol Phys. 2008; 72(4):1215-20.

5. Roeder F. Neoadjuvant radiotherapeutic strategies in pancreatic cancer. World J Gastrointest Oncol. 2016;89(4):830-8.

6. Shinoto M, Yamada S, Yasuda S, Imada H, Shioyama Y, Honda H, et al. Phase 1 trial of preoperative, short-course carbon-ion radiotherapy for patients with resectable pancreatic cancer. Cancer. 2013;119(1):45-51.

7. Dreher C, Habermehl D, Jäkel O, Combs SE. Effective radiotherapeutic treatment intensification in patients with pancreatic cancer: higher doses alone, higher RBE or both? Radiat Oncol. 2017;12(1):203.

8. Terashima K, Demizu Y, Hashimoto N, Jin D, Mima M, Fujii O, et al. A phase I/II study of gemcitabine-concurrent proton radiotherapy for locally advanced pancreatic cancer without distant metastasis. Radiother Oncol. 2012;103(1):25-31.

9. Hong TS, Ryan DP, Borger DR, Blaszkowsky LS, Yeap BY, Ancukiewicz M, et al. A phase $1 / 2$ and biomarker study of preoperative short course chemoradiation with proton beam therapy and capecitabine followed by early surgery for resectable pancreatic ductal adenocarcinoma. Int J Radiat Oncol Biol Phys. 2014;89(4):830-8.

10. Durante M, Tommasino F, Yamada S. Modeling combined chemotherapy and particle therapy for locally advanced pancreatic cancer. Front Oncol. 2015;5:145.

11. Knybel L, Cvek J, Otahal B, Jonszta T, Molenda L, Czerny D, et al. The analysis of respiration-induced pancreatic tumor motion based on reference measurement. Radiat Oncol. 2014;9:192.

12. Lomax AJ, Böhringer T, Bolsi A, Coray D, Emert F, Goitein G, et al. Treatment planning and verification of proton therapy using spot scanning: initial experiences. Med Phys. 2004;31(11):3150-7.

13. Phillips MH, Pedroni E, Blattmann H, Boehringer T, Coray A, Scheib S, et al. Effects of respiratory motion on dose uniformity with a charged particle scanning method. Phys Med Biol. 1992;37(1):223-33.

14. Bert C, Grözinger SO, Rietzel E. Quantification of interplay effects of scanned particle beams and moving targets. Phys Med Biol. 2008;53(9):2253-65.

15. Knopf A, Hong TS, Lomax A. Scanned proton radiotherapy for mobile targets - the effectiveness of re-scanning in the context of different treatment planning approaches and for different motion characteristics. Phys Med Biol. 2011;56(22):7257-71.

16. Zhang Y, Boye D, Tanner C, Lomax AJ, Knopf A. Respiratory liver motion estimation and its effect on scanned proton beam therapy. Phys Med Biol. 2012;57(7):1779-95.

17. Seco J, Robertson D, Trofimov A, Paganetti H. Breathing interplay effects during proton beam scanning: simulation and statistical analysis. Phys Med Biol. 2009:54(14):283-94.

18. Zenklusen SM, Pedroni E, Meer D. A study on repainting strategies for treating moderately moving targets with proton pencil beam scanning at the new gantry 2 at PSI. Phys Med Biol. 2010;55(17):5103-21.

19. Zhang Y, Huth I, Wegner M, Weber DC, Lomax AJ. An evaluation of rescanning technique for liver tumour treatments using a commercial PBS proton therapy system. Radiother Oncol. 2016;121(2):281-7.

20. Sarudis S, Karlsson Hauer A, Nyman J, Bäck A. Systematic evaluation of lung tumor motion using four-dimensional computed tomography. Acta Oncol. 2017;56(4):525-30

21. Abbas A, Chang B, Chen Z. Motion management in gastrointestinal cancers. J Gastrointest Oncol. 2014;5(3):223-35.

22. Boye D, Lomax AJ, Knopf A. Mapping motion from 4D-MRI to 3D-CT for use in 4D dose calculations: a technical feasibility study. Med Phys. 2013;40(6):061702.

23. Dolde K, Naumann P, Dávid C, et al. 4D dose calculation for PBS proton therapy of pancreatic cancer using repeated 4DMRI datasets. PMB. 2018; https://doi.org/10.1088/1361-6560/aad43f.

24. Batista V, Richter D, Chaudhri N, Naumann P, Herfarth K, Jäkel O. Significance of intra-fractional motion for pancreatic patients treated with charged particles. Radiat Oncol. 2018;13:120.

25. Landberg T, Chavaudra J, Dobbs J, Hanks G, Johansson KA, Möller T et al. Report 50, Journal of the International Commission on Radiation Units and Measurements. Volume os26, Issue 1, 1 September 1993, Pages NP, https:// doi.org/10.1093/jicru/os26.1.Report50. 
26. Rank CM, Heußer T, Buzan MT, Wetscherek A, Freitag MT, Dinkel J, et al. 4D respiratory motion-compensated image reconstruction of free-breathing radial MR data with very high Undersampling. Magn Reson Med. 2017;77(3):1170-83.

27. Thirion J. Image matching as a diffusion process: an analogy with Maxwell's demons. Med Image Anal. 1998;2(3):243-60.

28. Vanderstraeten B, Chin PW, Fix M, Leal A, Mora G, Reynaert N, et al. Conversion of $\mathrm{CT}$ numbers into tissue parameters for Monte Carlo dose calculations: a multi-centre study. Phys Med Biol. 2007;52(3):539-62.

29. Zhang Y, Knopf A, Tanner C, Lomax AJ. Online image guided tumour tracking with scanned proton beams: a comprehensive simulation study. Phys Med Biol. 2014;59(24):7793-817.

30. Bland JM, Altman DG. Correlation, regression and repeated observations: part 1 - correlation within subjects. Br Med J. 1995;310:446.

31. Bland JM, Altman DG. Correlation, regression and repeated observations: part 2 - correlation within subjects. Br Med J. 1995;310:633.

32. Shinoto M, Shigeru Y, Shigeo Y, et al. Phase 1 trial of preoperative, shortcourse carbon-ion radiotherapy for patients with Resectable pancreatic Cancer. Cancer. 2013;119(1):45-51.

33. Heerkens $H$, Reerink $O$, Intven MPW, Hiensch RR, van den Berg CAT, Crijns SPM, et al. Pancreatic tumor motion reduction by use of a custom abdominal corset. Phys Imaging Radiat Oncol. 2017;2:7-10.

34. Dolde K, Dávid C, Echner G, Floca R, Hentschke C, Maier F, et al. 4DMRIbased analysis of inter- and intrafractional pancreas motion and deformation with different immobilization devices. Biomed Phys Eng Express. 2019:5:025012. https://doi.org/10.1088/2057-1976/aaf9ae.

35. Dowdell S, Grassberger C, Sharp GC, Paganetti H. Interplay effects in proton scanning for lung: a 4D Monte Carlo study assessing the impact of tumor and beam delivery parameters. Phys Med Biol. 2013;58(12):4137-56.

36. Heerkens $H D$, van Vulpen $M$, van den Berg CA, Tijssen RH, Crijns SP, Molenaar IQ, et al. MRI-based tumor motion characterization and gating schemes for radiation therapy of pancreatic cancer. Radiother Oncol. 2014;111(2):252-7.

37. Heath E, Seuntjens J. A direct voxel tracking method for four-dimensional Monte Carlo dose calculations in deforming anatomy. Med Phys. 2006;33(2):434-45.

38. Yan C, Hugo G, Salquero FJ, Saleh-Sayah N, Weiss E. Sleeman WC. A method to evaluate dose errors introduced by dose mapping processes for mass conserving deformations. Med Phys. 2011;39(4):2119-28.

39. Rietzel $\mathrm{E}$, Chen GT. Deformable registration of $4 \mathrm{D}$ computed tomography data. Med Phys. 2006;33(11):4423-30.

40. Heath E, Collins DL, Keall PJ, Dong L, Seuntjens J. Quantification of accuracy of the automated nonlinear image matching and anatomical labeling "ANIMAL" nonlinear registration algorithm for 4D CT images of lung. Med Phys. 2007;34(11):4409-21

41. Horst E, Micke O, Moustakis C, et al. Conformal therapy for pancreatic cancer: variation of organ position due to gastrointestinal distention-implications for treatment planning. Radiology. 2002;222:681-6.

42. Li T, Schreibmann E, Thorndyke B, Tillman G, Boyer A, Koong A, et al. Radiation dose reduction in four-dimensional computed tomography. Med Phys. 2005;32(12):3650-60.

43. Lens E, van der Horst A, Kroon PS, van Hooft JE, Dávila Fajardo R, Fockens P, et al. Differences in respiratory-induced pancreatic tumor motion between $4 \mathrm{D}$ treatment planning $\mathrm{CT}$ and daily cone beam $\mathrm{CT}$, measured using intratumoral fiducials. Acta Oncol. 2014;53(9):1257-64.

44. Steitz J, Naumann P, Ulrich S, Haefner MF, Sterzing F, Oelfke U, et al. Worst case optimization for interfractional motion mitigation in carbon ion therapy of pancreatic cancer. Radiat Oncol. 2016;1 1(1):134.

45. Keall $P$, Mageras G, Balter J, et al. The management of respiratory motion in radiation oncology report of AAPM task group 76. Med Phys. 2006:33(10):3874-900.

46. Lagendijk JJW, Raaymakers BW, Raaijmakers AJE, et al. MRI/linac integration. Radiother Oncol. 2008;86(1):25-9.

47. Mutic S, Dempsey JF. The ViewRay system: magnetic resonance-guided and controlled radiotherapy. Semin Radiat Oncol. 2014;24(3):196-9.

48. Fallone BG. The rotating biplanar linac-magnetic resonance imaging system. Semin Radiat Oncol. 2014;24(3):200-2.

\section{Ready to submit your research? Choose BMC and benefit from:}

- fast, convenient online submission

- thorough peer review by experienced researchers in your field

- rapid publication on acceptance

- support for research data, including large and complex data types

- gold Open Access which fosters wider collaboration and increased citations

- maximum visibility for your research: over $100 \mathrm{M}$ website views per year

At BMC, research is always in progress.

Learn more biomedcentral.com/submissions 